\title{
Pushing the Measuring Capabilities of Silicon Drift Detectors for EDX Imaging of Low-Z Materials Down to Lithium
}

Adrian Niculae ${ }^{1}$, Thiago Barros ${ }^{1}$, Alois Bechteler ${ }^{1}$, Kathrin Hermenau ${ }^{1}$, Klaus Heinzinger ${ }^{2}$, Andreas Liebel $^{1}$, Heike Soltau ${ }^{1}$ and Lothar Strüder ${ }^{3}$

1. PNDetector GmbH, München, Germany.

2. PNSensor GmbH, München, Germany.

3. University of Siegen, Department of Physics, Siegen, Germany.

* Corresponding author: adrian.niculae@pndetector.de

Recent developments in the field of new materials and composites have been pushing for more powerful instrumentation in electron microscopy (EM). Besides the excellent spatial resolution needed to resolve nanoscale structures, the analytical capabilities of the instrument for revealing the composition are also very important.

Energy Dispersive X-ray spectroscopy (EDX) is one of the commonly used techniques for determining the elemental composition of the EM samples. Nowadays, almost every EDX system contains a Silicon Drift Detector (SDD) as X-ray sensing element. As a pioneer in the development and manufacturing of SDDs for EDX, we are continuously pushing the performance values of the SDDs towards the physical limits. Extending the detection limits towards light elements as low as Beryllium (108 eV) or Lithium $(54 \mathrm{eV})$ has become one of the major development tasks in the last years.

When it comes to detect extremely low energy X-rays like e.g. in the range 50 to $100 \mathrm{eV}$, the detector has to fulfill some conditions. First of all, a windowless detector is mandatory, any external window in the front of the sensor would substantially absorb low energy X-ray photons for which the emission rate from the sample is anyhow quite low.

Secondly the electronic noise contribution of the detector must be reduced to very low levels. Fig.1a shows the calculated energy resolution of a detector as Full Width Half Maximum (FWHM) as a function of the incoming photon energy. Whereas at higher photon energies the so-called Fano or statistical noise is the dominant component in the energy resolution, for very low energies the detector electronic noise becomes the dominant term and must be thus reduced as much as possible. In order to be able to detect $54 \mathrm{eV}$ X-rays, a noise level well below $30 \mathrm{eV}$ in FWHM is necessary.

Owing the integration of the first FET on chip-level, the total detector input capacitance can be reduced to levels as low as $50 \mathrm{fF}$ for detectors from the SD3 ${ }^{\text {plus }}$ series. Combined with very low leakage current technology an electronic noise level below $20 \mathrm{eV}$ FWHM is being achieved for such sensors. Fig. 1b shows the EDX spectrum from a Carbon sample measured with a SD3 ${ }^{\text {plus }}$ sensor in a windowless configuration.

The third ingredient which is needed for optimum detection of very light elements is a loss-free detector entrance window (that means the very thin passive layer on the SDD sensor). With the pnWindow technology, incomplete charge collection due to the loss in the entrance window is reduced to a minimum level, while insuring a gaussian-shape of the light element peaks. 
Fig.2 shows EDX spectra of low energy X-rays measured with a SDD $^{\mathrm{GL}}$ sensor with an intrinsic electronic noise of $20 \mathrm{eV}$ FWHM. The Al-L peak (Fig. 2a) is well separated from the noise peak, as is the Li-K peak@54 eV in Fig. 2b.

In this contribution we will present the analytical capabilities of SDD sensors of various types and sizes with regards to detecting very low energy x-ray photons in SEM applications. EDX images and spectra together with SEM images of selected samples will be shown and analyzed.
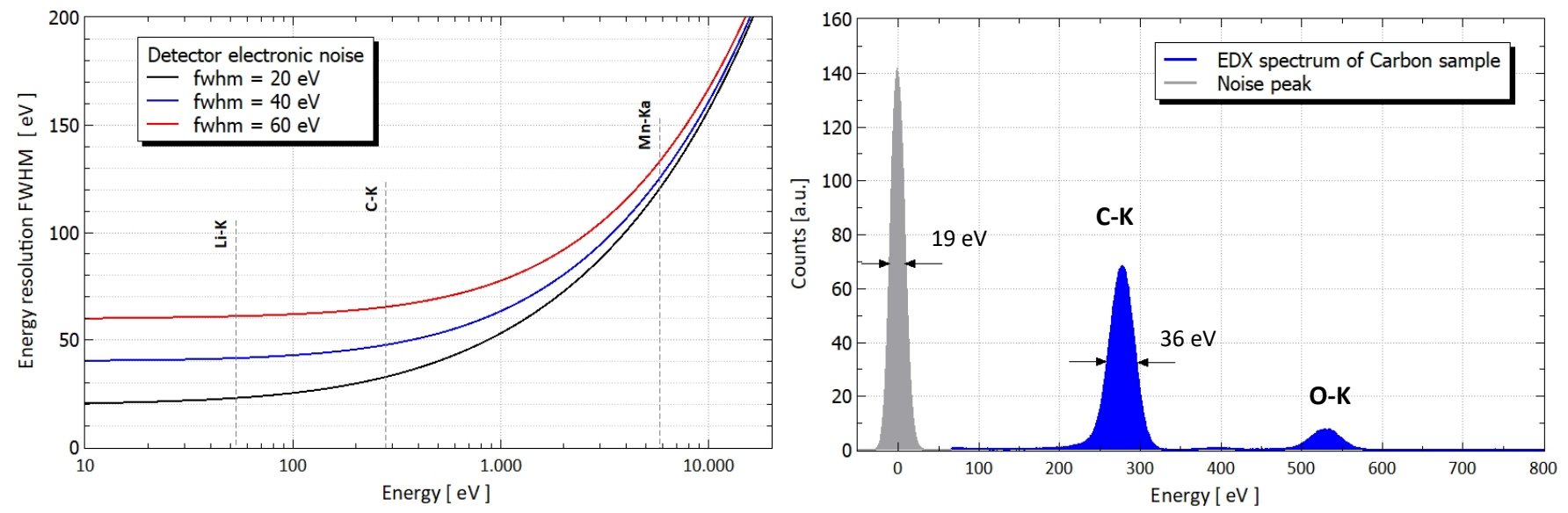

Figure 1. (a) Energy resolution of the detector as a function of incoming photon energy for different values of the detector intrinsic noise (given as FWHM); (b) EDX spectrum of a carbon sample measured with a detector having a noise level of $19 \mathrm{eV}$ in FWHM.
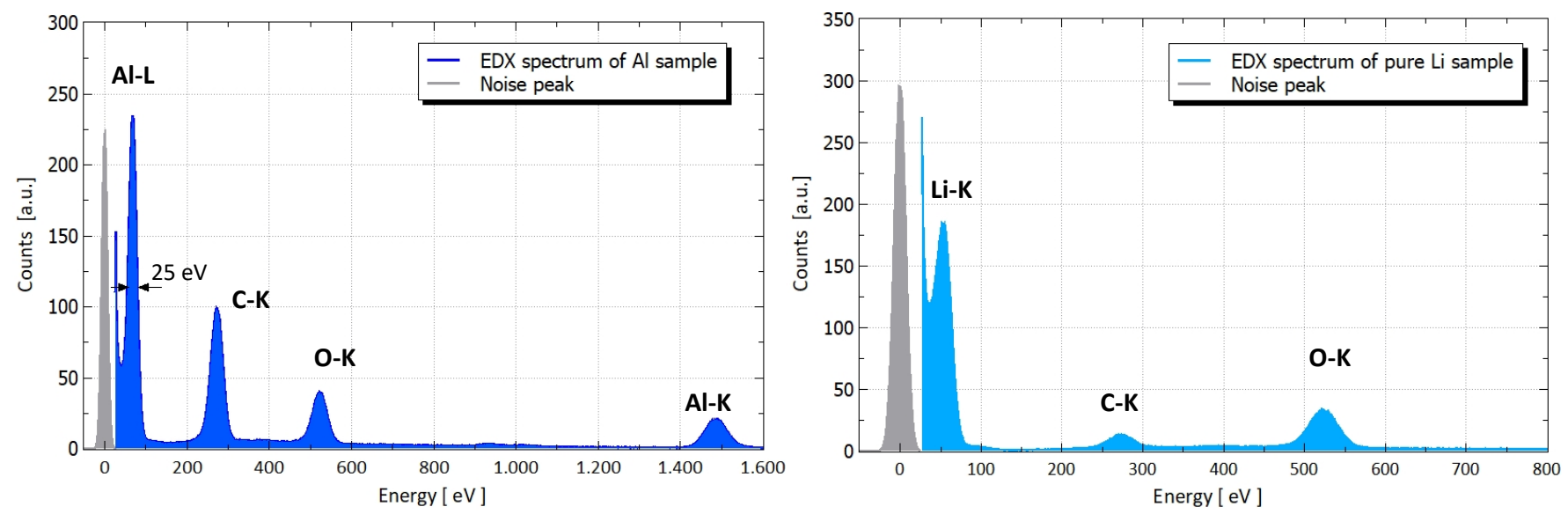

Figure 2. EDX spectra measured in an SEM from (a) Al sample showing the Al-L line at $68 \mathrm{eV}$ and (b) pure Lithium sample showing the Li-K at $54 \mathrm{eV}$ peak which can be clearly distinguished from the noise. 